\title{
Pain, in the boondocks
}

\author{
Saifee Rashiq, MB
}

Published online: 28 April 2010

(C) Canadian Anesthesiologists' Society 2010

\section{"What I object to is that you automatically treat me like an inferior...." Dennis the Peasant, Monty Python and the Holy Grail.}

The pathogenesis of neuropathic pain, and, by inference, mechanistic explanations of its effective treatment were once thought to follow a strict hierarchy. It was thought that structures and arrangements of increasing complexity and importance were encountered when moving from the periphery of the brain towards its centre, culminating in the fantastic subtleties of the cerebral cortex where impulses become feelings. The brain was considered the High Priest, the spinal cord the Grand Vizier, and so on down to the lumpen peripheral nociceptor, the merest hewer and drawer of neurologic wood and water whose job it was simply to fire when told to do so and leave the clever stuff to those up the line.

No longer. Convincing evidence has accumulated suggesting that peripheral neuronal structures actively participate in the modulation of both ascending and descending pain-related signals. ${ }^{1}$ This kind of knowledge is particularly exciting to clinicians, because it buttresses the practice (often commenced empirically) of using ad hoc topical preparations of systemic agents for the treatment of neuropathic pain in the hope of decreasing systemic side effects.

In this issue of the Journal, Park et al. ${ }^{2}$ present an elegant laboratory study in which the subcutaneous injection of pregabalin is seen to attenuate nociceptive behaviour in rats that had been subjected previously to unilateral sciatic nerve ligation, thus creating a standard model of neuropathic pain.

\section{S. Rashiq, MB ( $ه)$}

Department of Anaesthesiology and Pain Medicine,

University of Alberta, 8-120 Clinical Science Building,

Edmonton, AB T6G 2G3, Canada

e-mail:srashiq@ualberta.ca
The investigators were careful to create a cohort of animals that indeed demonstrated allodynia to light touch following surgical ligation of the sciatic roots. They then injected saline or different concentrations of pregabalin into the ipsilateral paw. This paw was then subjected to a series of standard challenges (heat, cold, pressure), and a dose-dependent reduction in nociceptive behaviour was observed.

Confounding factors were addressed in a logical and orderly fashion. Was this result merely indicative of the mechanical protective effect against the nociceptive stimulus of the $50 \mu \mathrm{L}$ fluid bolus and /or any attendant swelling or hemorrhage that might have occurred in the paw? No, because the investigators used a saline control group in which the attenuation was not seen. Could the result have been due to steadily increasing doses of pregabalin being absorbed systemically from the paw? No, because every rat also received the highest dose of pregabalin in the contralateral paw. Could it be that this result was simply the effect of dose-dependent sedation caused by pregabalin, resulting in the progressive inability of the animals to respond to the stimulus despite having the same amount of nociception? Unlikely, since the animals were also subjected to a standard test of gait stability and co-ordination and only showed such an effect at the very highest dose employed. All in all, this is high quality laboratory work that is readily meaningful to those of us who treat neuropathic pain. My only quarrel with the investigators is that they chose to test pregabalin instead of the inexpensive offpatent pain clinic workhorse, gabapentin.

Enthusiasm for the use of topically applied analgesics (as distinct from topically delivered systemic analgesics) has come and gone. There is evidence for efficacy of several of them, ${ }^{3}$ including capsaicin, non-steroidal antiinflammatory drugs, the lidocaine patch—sadly, unavailable in Canada, and doxepin-also sadly, no longer 
available in Canada as a commercial topical preparation. While recognizing that injecting a substance subcutaneously to achieve a local effect is not the same as rubbing it onto the skin, this study offers a sound rationale for conducting trials of peripheral preparations of pregabalin in clinical neuropathic pain. In all likelihood, the most practical approach would be to use creams or patches rather than the injections tested here. Such interventions would probably be very safe and certainly safer than many of the interventions we currently use for neuropathic pain. This wasn't a trial of pre-emptive analgesia because the nerve injury was already established before the pregabalin was administered, but perhaps there might be value in studying it for that purpose. Might the application of pregabalin cream on the night before thoracotomy, for instance, help prevent postoperative acute and chronic pain?

Clinical pain researchers who are motivated to explore the use of topical pregabalin further will want to begin with open-label dose-finding studies focusing on safety. It will be essential to use utmost care when reporting the way in which the drug is compounded for topical application, since idiosyncratic pharmaceutical practices can materially affect the way in which such drugs are delivered. ${ }^{3}$ There are many potential ways in which topical pregabalin could be tested, such as diabetic neuropathy, post herpetic neuralgia, and post-surgical neuropathy. However, the investigator should focus on recording clinically meaningful outcome measures and should not worry excessively about the generalizability of the particular disease state in which pregabalin is to be tested.

After all, it's only a model.

\section{La douleur hors des sentiers battus}

«Ce que je réprouve, c'est votre manière de me traiter comme un inférieur...» Dennis le paysan, Monty Python: Sacré Graal!

On a déjà pensé que la pathogenèse de la douleur neuropathique et, par conséquent, les explications mécanistes des traitements efficaces suivaient une hiérarchie stricte. On a dit que des structures et des configurations d'une complexité et d'une importance grandissantes sont découvertes au fur et à mesure qu'on passe de la périphérie du cerveau à son centre, pour culminer aux subtilités extraordinaires du cortex cérébral, où les impulsions se transforment en sensations. Le cerveau a été considéré comme le grand prêtre, la moelle épinière, comme le grand vizir et ainsi de suite jusqu'à l'informe nocicepteur périphérique, le plus simple tailleur et façonneur de la forêt et de l'eau cérébrales dont le travail consiste tout bonnement à tirer lorsqu'on lui ordonne de le faire et à laisser les éléments ingénieux à ceux qui se trouvent en haut de l'échelle.

Ce n'est plus le cas. On a amassé des preuves convaincantes qui portent à croire que les structures neuronales périphériques participent activement à la modulation des signaux liés à la douleur, tant ascendants que descendants. ${ }^{1}$ Ce type de connaissance est emballant, en particulier pour les cliniciens puisqu'il appuie la pratique (souvent amorcée sur une base empirique) qui consiste à utiliser $a d$ hoc des préparations topiques d'agents systémiques pour le traitement de la douleur neuropathique en vue de diminuer les effets secondaires systémiques.

Dans le présent numéro du Journal, Park et al. ${ }^{2}$ présentent une étude de laboratoire bien réalisée dans le cadre de laquelle on a constaté que l'injection sous-cutanée de prégabaline atténue le comportement nociceptif chez les rats ayant précédemment subi une ligature unilatérale du nerf sciatique, créant ainsi un modèle standard de douleur neuropathique. Les chercheurs ont pris soin de former une cohorte d'animaux présentant une allodynie à l'effleurement à la suite de la ligature chirurgicale des racines sciatiques. Ils ont ensuite injecté une solution physiologique ou diverses concentrations de prégabaline dans la patte ipsilatérale, puis ils ont exposé cette patte à une série de conditions standards (chaleur, froid, pression). Une réduction du comportement nociceptif en fonction de la dose a été observée.

Les facteurs confondants ont été examinés de façon logique et méthodique. Ce résultat illustre-t-il simplement l'effet protecteur mécanique des $50 \mu \mathrm{L}$ de liquide injecté contre le stimulus nociceptif et/ou toute enflure ou hémorragie concomitante qui aurait pu survenir dans la patte? Non, car les chercheurs ont injecté une solution physiologique au groupe témoin, et aucune réduction n'a été constatée. Est-ce que le résultat peut être attribuable aux doses de prégabaline régulièrement augmentées qui ont été systématiquement absorbées à partir de la patte? Non, car chaque rat a également reçu la plus forte dose de prégabaline dans la patte contralatérale. Est-ce que ce résultat illustre simplement l'effet sédatif de la prégabaline en fonction de la dose, qui empêche graduellement les animaux de réagir au stimulus malgré un degré de nociception inchangé? C'est improbable, car les animaux ont également passé un test standard de stabilité de la démarche et de la coordination et qu'un tel effet a été constaté uniquement avec la plus forte dose utilisée. Tout bien considéré, il s'agit d'un travail de laboratoire de grande qualité dont la pertinence est évidente pour ceux d'entre nous qui traitent la douleur neuropathique. Mon seul différend avec les chercheurs, c'est qu'ils ont choisi de tester la prégabaline plutôt que la classique gabapentine, analgésique clinique générique et bon marché.

L'engouement pour des analgésiques en application locale (et non les analgésiques systémiques topiques) est 
passé. L'efficacité de certains de ces analgésiques est prouvée, $^{3}$ notamment celle de la capsaïcine, des anti-inflammatoires non stérö̈diens, des timbres de lidocaïne - qui ne sont malheureusement pas offerts au Canada - et de la doxépine — qui n'est malheureusement plus offerte au Canada en tant que préparation topique commerciale. Même en admettant que l'injection sous-cutanée d'une substance pour obtenir un effet local diffère de son application sur la peau, l'étude fournit de bonnes raisons de mener des essais sur les préparations périphériques de prégabaline dans le domaine de la douleur neuropathique clinique. Selon toute probabilité, l'approche la plus pratique consisterait à utiliser des crèmes ou des timbres plutôt que les injections testées dans le cas présent. De telles interventions seraient probablement très sécuritaires, voire certainement plus sécuritaires qu'un grand nombre des interventions que nous utilisons actuellement pour le traitement de la douleur neuropathique. Il ne s'agit pas d'un essai portant sur l'analgésie préventive, puisque la lésion nerveuse a été établie avant l'administration de la prégabaline; il pourrait toutefois être utile de mener une étude sur le sujet. Par exemple, l'application de crème de prégabaline en soirée la veille d'une thoracotomie peut-elle contribuer à prévenir les douleurs postopératoires aiguës et chroniques?

Les chercheurs cliniciens intéressés par la douleur qui veulent étudier davantage l'utilisation de prégabaline topique souhaiteront commencer par des études ouvertes impliquant plusieurs doses et axées sur l'innocuité. Il sera essentiel de décrire avec le plus grand soin la composition du médicament en application topique, car les pratiques pharmaceutiques idiosyncratiques peuvent avoir un effet significatif sur la façon dont de tels médicaments sont absorbés. ${ }^{3}$ Il y a plusieurs façons dont la prégabaline pourrait être étudiée, notamment dans les domaines de la neuropathie diabétique, de l'algie post-zostérienne et de la neuropathie postopératoire. Le chercheur devrait toutefois se concentrer sur la cueillette de résultats cliniques pertinents sans trop se préoccuper de savoir si les conclusions obtenues dans la maladie pour laquelle la prégabaline est étudiée en particulier peuvent être appliquées à d'autres situations.

Après tout, il s'agit seulement d'un modèle.

Competing interests None declared.

\section{References}

1. Costigan M, Scholz J, Woolf CJ. Neuropathic pain: a maladaptive response of the nervous system to damage. Annu Rev Neurosci 2009; 32: 1-32.

2. Park HJ, Joo HS, Chang HW, et al. Attenuation of neuropathyinduced allodynia following intraplantar injection of pregabalin. Can J Anesth 2010; 57. doi:10.1007/s12630-010-9318-0.

3. de Leon-Casasola OA. Multimodal approaches to the management of neuropathic pain: the role of topical analgesia. J Pain Symptom Manage 2007; 33: 356-64. 